\title{
Spatio-Temporal Polar-Inclined Space Mission Architecture for a Refined Retrieve of the Earth's Gravity Field
}

\author{
Basem Elsaka1,2 \\ ${ }^{1}$ Institute of Geodesy and Geoinformation, University of Bonn, Bonn, Germany \\ ${ }^{2}$ National Research Institute of Astronomy and Geophysics (NRIAG), Cairo, Egypt \\ Email: elsaka@geod.uni-bonn.de
}

How to cite this paper: Elsaka, B. (2019) Spatio-Temporal Polar-Inclined Space Mission Architecture for a Refined Retrieve of the Earth's Gravity Field. World Journal of Engineering and Technology, 7, 640-651. https://doi.org/10.4236/wjet.2019.74047

Received: November 5, 2019

Accepted: November 23, 2019

Published: November 26, 2019

Copyright $\odot 2019$ by author(s) and Scientific Research Publishing Inc. This work is licensed under the Creative Commons Attribution-NonCommercial International License (CC BY-NC 4.0). http://creativecommons.org/licenses/by-nc/4.0/ (c) (i) \& Open Access

\begin{abstract}
Future satellite gravity missions (FGMs) have been intensively studied during the last recent years for the era beyond the successful previous GRACE and current GRACE Follow-on satellite missions. Previous studies have investigated the gravity field recovery derived from combined two satellite-pairs (referred here as PI-FGM, a single polar satellite-pair like the GRACE mission combined with another inclined satellite-pair) with different orbital heights of few kilometers and different repeat orbital periods. In this contribution, new innovative idea is introduced by designing the inclined satellite-pair of the FGM at the same orbital height of the polar-type with shifted spatio-temporal (ST-FGM) orbital parameters to avoid any possible collision risk between the two satellite-pairs, polar and inclined, of the FGM architecture. The repeat orbits issue will be taken into consideration through the manuscript and will be set as identical as possible for a fair comparison. The findings through a full-scale simulation analysis show that the new design of shifted spatio-temporal polar-inclined (ST-FGM) mission architecture basically outperforms the two satellite-pairs having different orbital heights (i.e. the PI-FGM mission configuration). Regarding the gravity field recovery, the ST-FGM architecture retrieves the geoid heights with standard deviations of about $17.0 \mathrm{~mm}$ providing more isotropic error distribution. An overall improvement by a factor of about 80 and 60 is provided by the ST-FGM and PI-FGM mission architectures, respectively, with respect to the GRACE-like formation and a factor of about 2.4 and 1.8, respectively, with respect to the smoothed gravity solution using the Gaussian filter at radius $400 \mathrm{~km}$. Therefore, the shifted spatio-temporal polar-inclined (ST-FGM) is worthy recommended as stable mission architecture and would be considered as one of the future gravity missions.
\end{abstract}




\section{Keywords}

Space Mission Architectures, Orbit Design, Repeat Orbit, Gravity Field Recovery, Full-Scale Simulation

\section{Introduction}

The new satellite technology is greatly appreciated because of the achieved high improved information of the Earth's processes and particularly global gravity field. High resolved gravity field recovery has been measured via four dedicated satellite missions; the challenging Mini satellite Payload (CHAMP) [1] launched between July 2000 and September 2010, the Gravity Recovery and Climate Experiment (GRACE) [2] launched from March 2002 until October 2017 and its successive (Follow-On) mission GRACE-FO [3] launched on 22 May 2018 and the Gravity field and steady-state Ocean Circulation Explore (GOCE) [4] launched between March 2009 and November 2013. However, still some restrictions exist which confine these successful missions as addressed by e.g. [5]. For instance, the key limitation of CHAMP was to derive higher spatial and temporal resolutions. For GOCE, although it achieved higher spatial resolution with higher static gravity field, it was a challenging issue to determine the temporal gravity variations due to the limited mission duration. According to the GRACE and its Follow-On mission (GRACE-FO) realizing the low-low satellite-to-satellite tracking concept, they only provide a single gravity field gradient component in the along-track direction given by the baseline between its two spacecrafts.

Correspondingly, a number of novel concepts using different configuration options toward a future gravity mission (FGM) have been investigated in the last years (e.g. [6]-[20]). Among of these studies, the global and regional gravity recovery derived from single satellite-pair (such as the GRACE and GRACE-FO designs) has been improved by the addition of another inclined satellite-pair.

Following the idea of supporting the FGM of polar-type mission with an inclined satellite-pair, new innovative idea is provided within this paper. Here, the inclined satellite-pair is shifted from the polar-type spatially through the orbital RAAN (Right Ascension of Ascending Nodes) angle and temporally through the orbital mean anomaly. This avoids any possible collision risk that takes place between both polar and inclined satellite-pairs. Despite other studies such as Wiese et al. [13] and Elsaka et al. [15] avoided the collision risk by putting both satellite-pairs at different orbital altitudes, however, due to the fact that the inclined satellite-pair has no altitude control, the collision risk is not impossible.

The spatio-temporal future gravity mission (ST-FGM) is characterized by the same orbital altitude of its spacecrafts, and hence, provides almost the same repeat periods of its four-satellites. In the following, full-scale simulation scenarios are given in order to retrieve the global Earth's gravity field from both the ST-FGM and the classical polar-inclined configurations (PI-FGM). 
The rest of this article is organized as follows: Section 2 describes the orbital design of the ST-FGM mission architecture. Full-scale simulation scenarios regarding the recovery of the gravitational field are presented in Section 3. The results and discussion are explained in Section 4. Finally, some conclusions are given in Section 5.

\section{Orbital Characteristics of PI-FGM and ST-FGM Space Architecture}

\subsection{Orbital Elements}

The orbital altitude for all simulated ST-FGM and PI-FGM satellites has been set up at $h=334 \mathrm{~km}$, except for the inclined satellite-pair of PI-FGM which was set up at $h=360 \mathrm{~km}$. In addition, a GRACE-type configuration has been used in this paper for comparison as a reference mission with a nominal orbital height of about $h=462 \mathrm{~km}$ similar to [15]. The polar orbit of both ST-FGM and PI-FGM has been actually set up at inclination $i=91^{\circ}$ based on the recent results and the recommendation of [20]. Nie et al. [20] showed that the formation flying at $i=$ $91^{\circ}$ basically outperforms the other orbital inclinations such as $87^{\circ}$ and $89^{\circ}$, especially in the medium- and low-latitude regions. Compared to latter inclinations, the orbital inclination $91^{\circ}$ has reduced about $22 \%$ of noise over the ocean area and about $17 \%$ over land areas, and hence, $i=91^{\circ}$ has been considered for the polar orbits of the current study.

The inclined orbits of ST-FGM and PI-FGM mission constellations (integrated at $i=75^{\circ}$ ) have been selected based on [13], who recommended the flying of an inclined satellite-pair besides polar one at degrees between $70^{\circ}$ and $75^{\circ}$ in order to avoid the collision risk between both polar and inclined satellite-pairs. However, since the inclined orbits of PI-FGM satellites have no altitude control, the collision risk is not impossible. Table 1 shows the orbital elements, semi-major axis (a), eccentricity (e), inclination $(i)$, RAAN angle $(\Omega)$, argument of perigee $(\Omega)$ and mean anomaly $(M)$, of the ST-FGM and PI-FGM satellites. In addition, Table 1 gives the selected orbital parameters for the "reference mission" GRACE-like formation for a comparison purpose.

The simulated GRACE-like scenario adopts here the details of the real GRACE mission with an orbital inclination of $89^{\circ}$, "initial" orbital height of $462 \mathrm{~km}$ and inter-satellite range of $220 \mathrm{~km}$ (see Table 1). Figure 1 shows the investigated mission architectures; ST-FGM and PI-FGM, in addition to the GRACE-like mission as a reference mission and their relative motion (pseudo-) initial reference frame between satellites given by XYZ axes in $\mathrm{km}$. The known GRACE-like configuration (Figure 1(a)) is characterized by simple collinear twin-satellite geometry whose observables are sensitive only in one single-arm (i.e. along-track) direction.

Although the other two ST-FGM (Figure 1(b)) and PI-FGM (Figure 1(c)) mission constellations collect observables in along-track flight direction, the sensitivity and isotropy are improved due to the cross-track flight direction 
Table 1. Orbital elements ( $a, e, i, \Omega, \omega, M$ ) of the ST-FGM and PI-FGM mission architectures with respect to the GRACE-like formation.

\begin{tabular}{cccccccc}
\hline \multirow{2}{*}{$\begin{array}{c}\text { Satellite } \\
\text { Architecture }\end{array}$} & \multicolumn{7}{c}{ Orbital Elements } \\
\cline { 2 - 7 } & Sat A & $6712.137(h=334 \mathrm{~km})$ & 0.001 & 91 & 0.0 & 0.0 & 0.0 \\
\hline \multirow{2}{*}{ PI-FGM } & Sat B & $6712.137(h=334 \mathrm{~km})$ & 0.001 & 91 & 0.0 & 0.0 & -0.84 \\
& Sat C & $6738.137(h=360 \mathrm{~km})$ & 0.001 & 75 & 0.0 & 0.0 & 0.0 \\
& Sat D & $6738.137(h=360 \mathrm{~km})$ & 0.001 & 75 & 0.0 & 0.0 & -0.84 \\
& Sat A & $6712.137(h=334 \mathrm{~km})$ & 0.001 & 91 & 0.0 & 0.0 & 0.0 \\
& Sat B & $6712.137(h=334 \mathrm{~km})$ & 0.001 & 91 & 0.0 & 0.0 & -0.84 \\
ST-FGM & Sat C & $6712.137(h=334 \mathrm{~km})$ & 0.001 & 75 & 0.4058 & 0.0 & 180.0 \\
& Sat D & $6712.137(h=334 \mathrm{~km})$ & 0.001 & 75 & 0.4058 & 0.0 & 179.16 \\
& Sat A & $6840.137(\mathrm{~h}=462 \mathrm{~km})$ & 0.001 & 89 & 0.0 & 0.0 & 0.0 \\
GRACE-like & Sat B & $6840.137(\mathrm{~h}=462 \mathrm{~km})$ & 0.001 & 89 & 0.0 & 0.0 & -1.68 \\
\hline
\end{tabular}

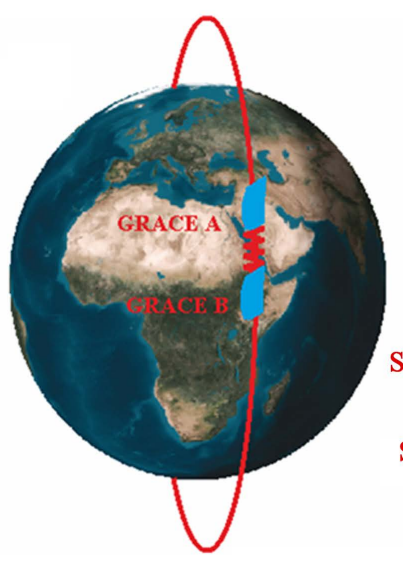

(a)

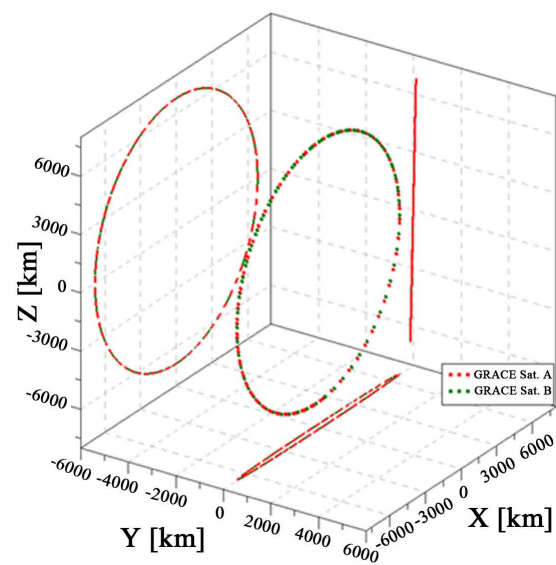

(d)

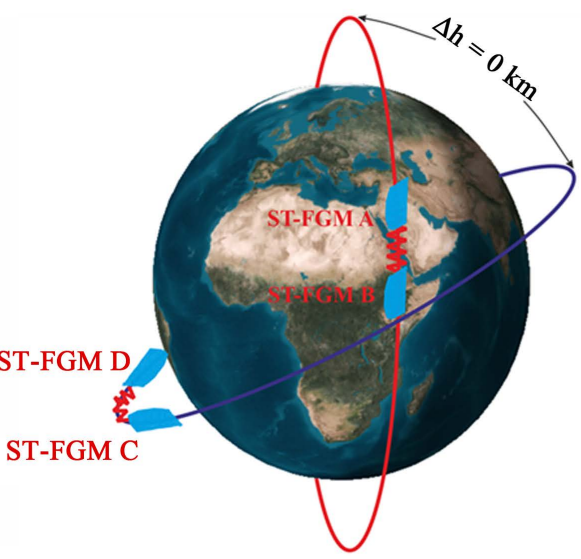

(b)

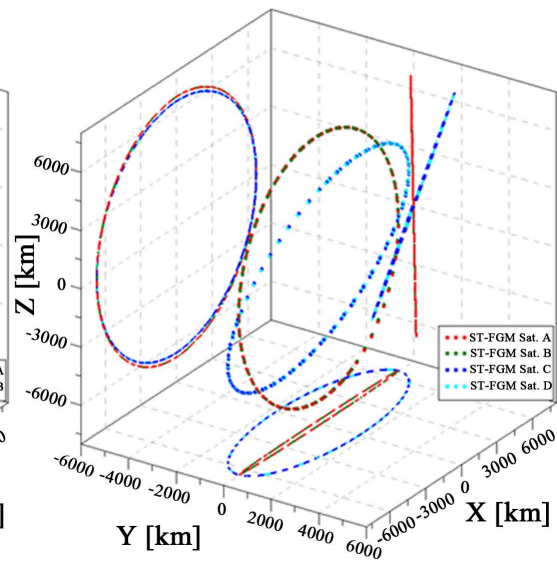

(e)

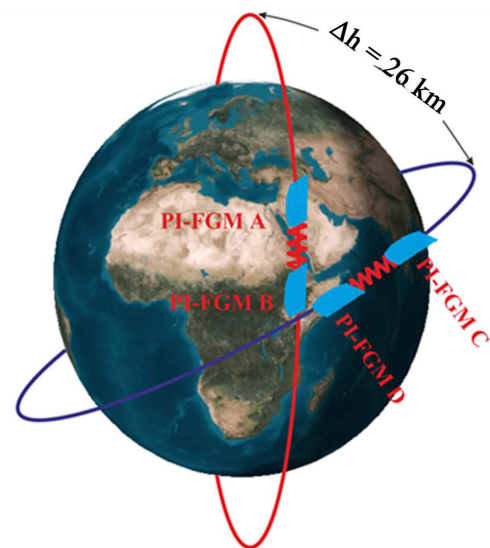

(c)

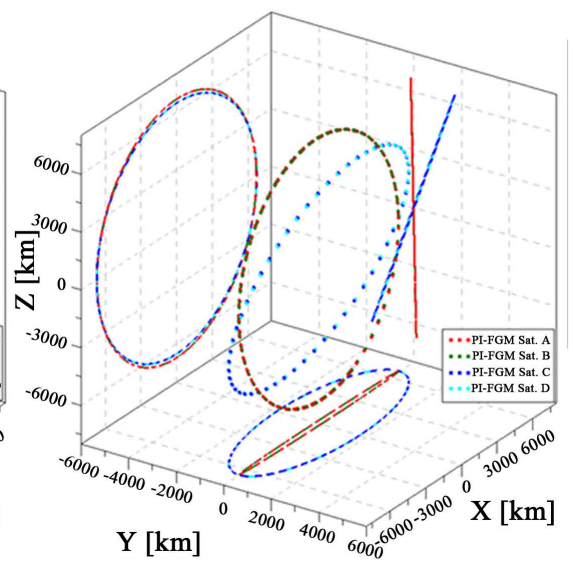

(f)

Figure 1. The investigated mission architectures; ST-FGM (b) and PI-FGM (c), in addition to the GRACE-like mission (a) as a reference mission and their relative motion in (pseudo-)initial reference frame given by XYZ axes in km ranging between \pm 6700 $\mathrm{km}, \pm 170 \mathrm{~km}$ and $\pm 6500 \mathrm{~km}$, respectively, except for Y-axis of satellites CD ranging between $\pm 1700 \mathrm{~km}$. 
provided by the inclined satellite-pair with respect to (w.r.t.) the polar satellite-pair as shown in the XY-plane of Figure 1(e) and Figure 1(f).

The inter-satellite baseline between satellites AB as well as CD of ST-FGM and PI-FGM mission configurations has been set to be approximately $100 \mathrm{~km}$ as shown in Figures 2(b)-(d), whereas the nominal inter-satellite range of GRACE-like has been set to $220 \mathrm{~km}$ (Figure 2(a)). The inter-satellite velocities of GRACE-like range between -0.7 to $0.4 \mathrm{~m} / \mathrm{s}$, of satellites AB of both ST-FGM and PI-FGM missions range between -0.3 to $0.2 \mathrm{~m} / \mathrm{s}$, of satellites $\mathrm{CD}$ of ST-FGM configuration range between $\pm 0.25 \mathrm{~m} / \mathrm{s}$ and of satellites CD of PI-FGM configuration range between -0.4 to $0.6 \mathrm{~m} / \mathrm{s}$. Furthermore, Figure $2(\mathrm{e})$ and Figure 2(f) display the inter-satellite distances (in meter) between satellites AC of both investigated mission architectures. As mentioned in the introductory section, the collision risk between both satellite-pairs (AB with $\mathrm{CD}$ ) of PI-FGM, which has no altitude control, is not impossible and/or can reach critical distances (see Figure 2(f)). Therefore, the idea of the current paper by designing the inclined satellite-pair (CD) with mean anomaly offset of about $\Delta \mathrm{M}=180^{\circ}$ (see Figure 1(b)) is very useful to avoid such critical relative motion and to keep it safe as shown in Figure 2(e).

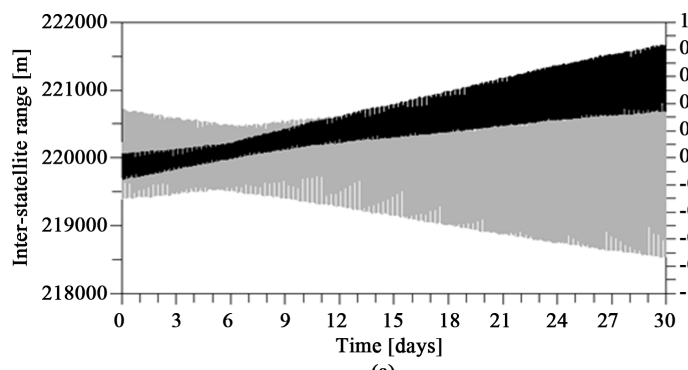

(a)

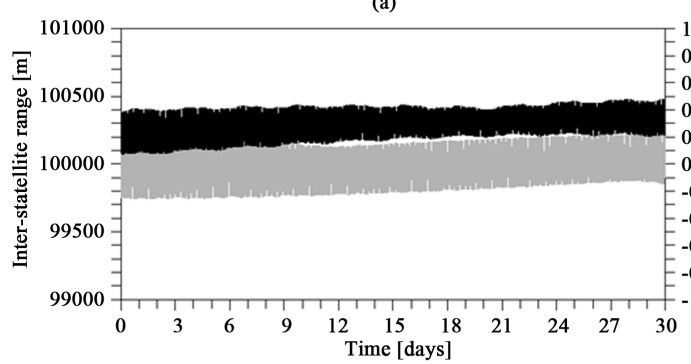

(c)

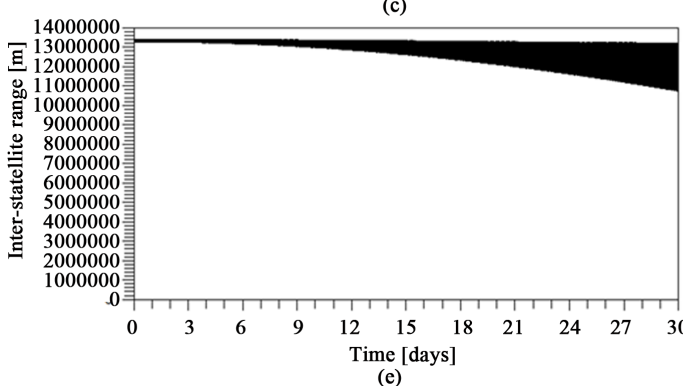

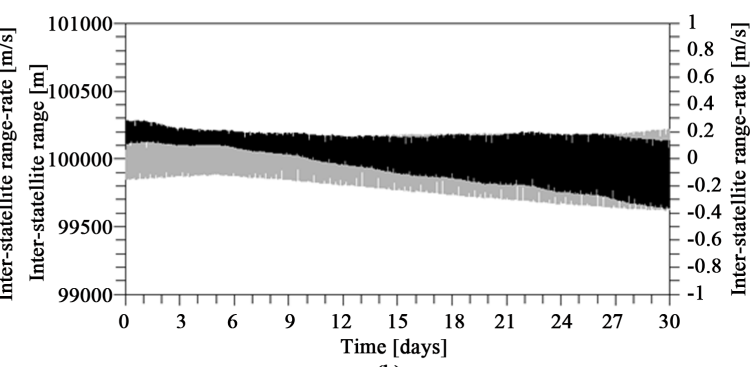

(b)

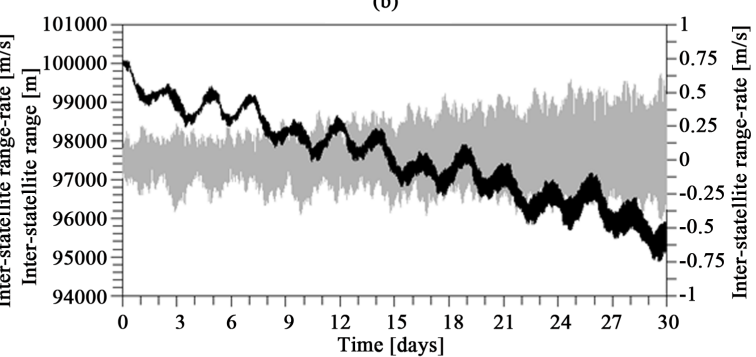

(d)

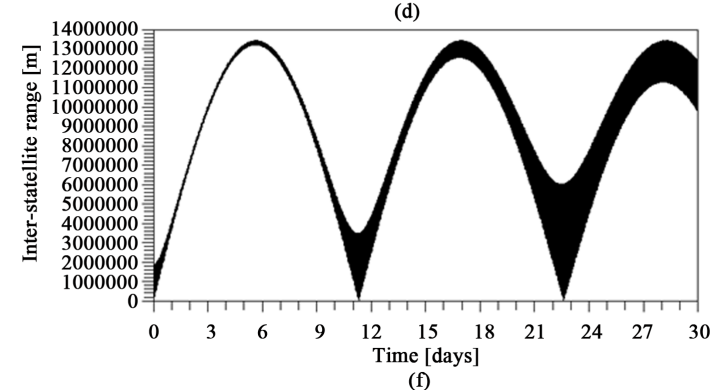

Figure 2. Inter-satellite range $[\mathrm{m}]$ (black curve on left y-axis) and range-rate $[\mathrm{m} / \mathrm{s}]$ (gray curve on right y-axis) of one month between satellites (a) AB of GRACE-like; (b) AB of ST-FGM and PI-FGM; (c) CD of ST-FGM; (d) CD of PI-FGM as well as between satellites (e) AC of ST-FGM and (f) AC of PI-FGM. 


\subsection{Selection of Repeat Orbits}

Considering the orbital elements given in Table 1, one finds that the orbital elements have been carefully selected to get repeated orbital period $\left(P_{n}\right)$ as same as possible, whose condition is given as [21]

$$
P_{n}=2 \pi \frac{\alpha}{\beta\left(\omega_{e}-\dot{\Omega}\right)}=\frac{2 \pi}{\dot{\omega}+\dot{M}} .
$$

where $\beta$ represents the number of orbits (i.e. nodal revolutions) after an integer number $(\alpha)$ of Earth rotations at time in nodal days, $\omega_{e}$ represents the Earth's angular velocity and the three terms $\dot{\Omega}, \dot{\omega}$, and $\dot{M}$ are secular changes of the orbital elements $\Omega, \omega$ and $M$ (see Table 1), respectively, which can be calculated according to [22] as

$$
\begin{gathered}
\frac{\mathrm{d} \Omega}{\mathrm{d} t}=\frac{3 n C_{20} R^{2}}{2 a^{2}\left(1-e^{2}\right)^{2}} \cos i, \\
\frac{\mathrm{d} \omega}{\mathrm{d} t}=\frac{3 n C_{20} R^{2}}{4 a^{2}\left(1-e^{2}\right)^{2}}\left(1-5 \cos ^{2} i\right), \\
\frac{\mathrm{d} M}{\mathrm{~d} t}=n-\frac{3 n C_{20} R^{2}}{2 a^{2}\left(1-e^{2}\right)^{3 / 2}}\left(3 \cos ^{2} i-1\right),
\end{gathered}
$$

where $n$ is the satellite's mean motion, $C_{20}$ is the second zonal term of the geopotential, $R$ is the Earth's radius, $a, e$ and $i$ are the orbital elements given in Table 1. The repeat period condition in Equation (1) is related to the mean motion of the satellite as

$$
n=\frac{\alpha}{\beta}\left(\omega_{e}-\dot{\Omega}\right)-(\dot{\omega}+\dot{M}) .
$$

After substituting the secular rates of Equation (2) into Equation (3), the ratio $\beta / \alpha$ can be easily computed as [9]

$$
n=\frac{\alpha}{\beta} \omega_{e}\left(1-\frac{3}{2} j_{2}\left[\frac{R}{a}\right]\left[4 \cos ^{2} i-\frac{\beta}{\alpha} \cos i-1\right]\right) .
$$

Since Equation (4) depends on the orbital altitude and inclination, the given parameters in Table 1 are selected to fairly have repeat orbits as identical as possible, which in this case are 11 days. Figure 3 shows the satellite orbital revolutions of all investigated mission configurations.

\section{Full-Scale Simulation Scenarios}

Two main numerical simulation steps, forward and backward, have been performed to generate the satellite observations and to analyze the gravity field of each formation over a time span of one month (January 2006 is selected here). The GROOPS (Gravity Recovery Object Oriented Programming System) software developed in the Institute of Geodesy and Geoinformation of the Bonn University (see [23]) has been used for both forward and backward steps. 


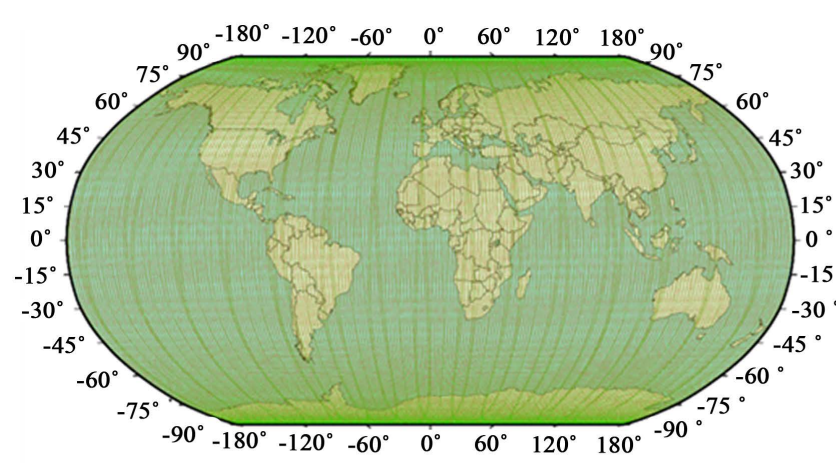

(a)

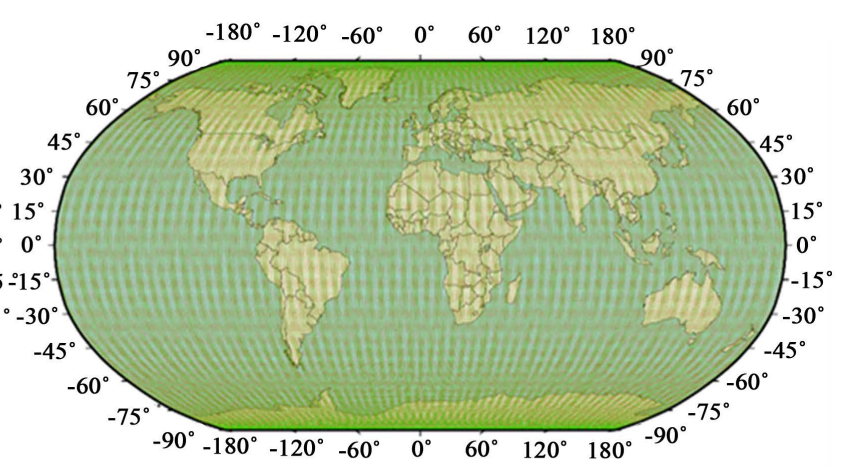

(b)

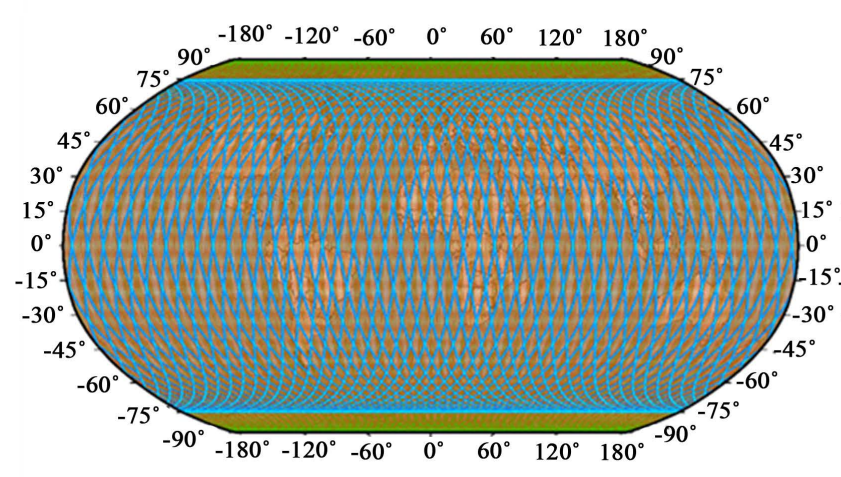

(c)

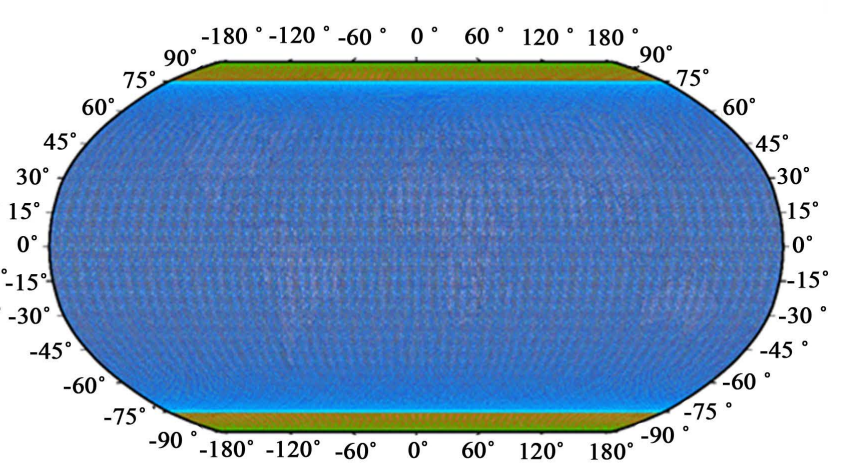

(d)

Figure 3. Satellite orbital revolutions of the investigated space mission architectures; (a) satellites AB of GRACE-like; (b) satellites $\mathrm{AB}$ of ST-FGM and PI-FGM; (c) satellites ABCD of ST-FGM and (d) satellites ABCD of PI-FGM.

Regarding to the forward step, the initial parameters for integrating satellite observations are already shown in Table 1. Table 2 shows the measurement noises added to the error-free observations performed in the forward step. A random Gaussian noise for contaminating the orbital velocities for all satellites with standard deviation (SD) equal to $0.2 \mu \mathrm{m} / \mathrm{s}$ has been generated. For inter-satellite baseline, a white noise in range-rate for GRACE of $0.21 \mu \mathrm{m} / \mathrm{s}$ and for the other satellites of $10 \mathrm{~nm} / \mathrm{s}$ was generated. We have to mention that the investigated configurations, except for GRACE, have been considered within this paper as drag-free missions, which don't carry accelerometer data; however, simulated accelerometer noise has been created of level $3 \times 10^{-10} \mathrm{~m} / \mathrm{s}^{2}$ assuming that this noise level is the error associated with the drag-free measurement. For the GRACE-like "reference" mission, the acceleration noise level of $9.8 \times 10^{-9} \mathrm{~m} / \mathrm{s}^{2}$ was applied as given in [15].

Regarding the backward (gravity analysis) step, the satellite observations (simulated in the first step) in terms of noisy kinematic orbits, noisy inter-satellite range-rate, noisy accelerometer and star-camera (for satellite attitude) of the STand PI-FGM architectures besides the GRACE scenario to estimate the gravity field parameters were used. The so-called "static scenario" is investigated, where satellite noisy measurements were set up and used in the observation equations to produce the static gravity field parameters in terms of spherical harmonics up to degree/order (d/o) 100/100. 
Table 2. Standard deviations (SD) of measurement noises used for the simulated observations of the investigated FGM space mission architectures vs GRACE-like formation.

\begin{tabular}{ccc}
\hline \multirow{2}{*}{ Noise } & \multicolumn{2}{c}{ Satellite Formation } \\
\cline { 2 - 3 } & GRACE-like & FGM Satellites \\
\hline Orbit Position & $0.20 \mu \mathrm{m} / \mathrm{s}$ & $0.20 \mu \mathrm{m} / \mathrm{s}$ \\
Inter-sat. range & $0.21 \mu \mathrm{m} / \mathrm{s}$ & $10.0 \mathrm{~nm} / \mathrm{s}$ \\
Accelerometer & $9.80 \mathrm{~nm} / \mathrm{s}$ & $0.30 \mathrm{~nm} / \mathrm{s}$ \\
\hline
\end{tabular}

\section{Results and Discussion}

The gravity field solutions determined from the investigated satellite configurations are given in the spectral domain within this section in terms of difference degree variances (DDV) of geoid heights. The DDV have been often used to quantify the powers of signal and error in the gravity field estimates as

$$
\sigma_{n}=\sqrt{\sum_{m=0}^{n} \Delta c_{n m}^{2}+\Delta s_{n m}^{2}}
$$

with $\Delta C_{n m}$ and $\Delta S_{n m}$ the differences between the estimated "recovered" gravity coefficients and the reference "true" model (ITG-GRACE2010s [24] in this case).

Moreover, the formal errors of the estimated gravity field solution have been obtained from the formula

$$
\delta \sigma_{n}=\sqrt{\sum_{m=0}^{n} \Delta \sigma_{C n m}^{2}+\Delta \sigma_{S n m}^{2}}
$$

with $\Delta \sigma_{C n m}$ and $\Delta \sigma_{S n m}$ representing the uncertainties in the estimation of the gravity coefficients $\Delta C_{n m}$ and $\Delta S_{n m}$, respectively. Figure 4 shows the difference degree variances $\left(\sigma_{n}\right)$ of geoid heights and their corresponding formal errors $\left(\delta \sigma_{n}\right)$ between the estimated spherical harmonics coefficients and the reference ones. Additionally, the results are plotted spatially in terms of geoid errors on Earth's maps as shown in Figure 5. The statistics of the geoid heights in terms of $\mathrm{SD}$, mean, minimum and maximum values are indicated in Table 3.

As seen from Figure 4, both retrieved gravity signals as detected from satellite observations of ST-FGM and PI-FGM mission architectures (see Figure 1(b) and Figure 1(c), respectively) outperform the GRACE-like gravity solution reaching approximately two full orders of magnitude in the spectral domain as well as the filtered GRACE-like solution using Gaussian Filter [25] at radius 400 $\mathrm{km}$. Furthermore, the retrieved gravity signal determined by ST-FGM mission constellation (Figure 1(b)) surpasses that one determined by the PI-FGM (Figure $1(\mathrm{c})$ ), especially at medium-to-short wavelength range of the gravity spectra (from d/o 50 onward). This improvement can be also spatially seen in Figure 5(c) in the middle parts of the Pacific Ocean, the Caribbean Sea and the western parts of the Indian Ocean. An overall SD of retrieved geoid heights of about $0.17 \mathrm{~cm}$ (Table 3 ) by the ST-FGM mission architecture provides improvements by a factor of about 80 w.r.t. the GRACE-like formation and a factor of $\approx 2.5$ w.r.t. the smoothed gravity solution using the Gaussian filter at radius $400 \mathrm{~km}$ (see Figure 5(b)). Whereas, in case of the PI-FGM gravity solution 


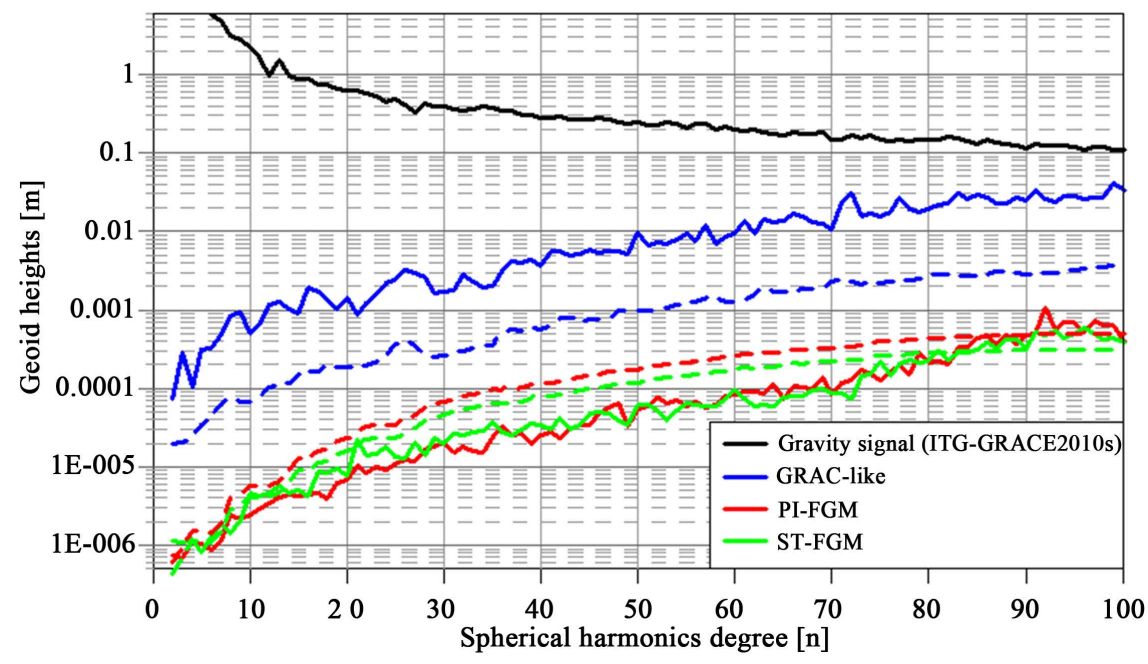

Figure 4. Gravity field solutions in terms of difference degree variance (solid lines) of geoid heights $[\mathrm{m}]$ and their corresponding formal errors (dashed lines).
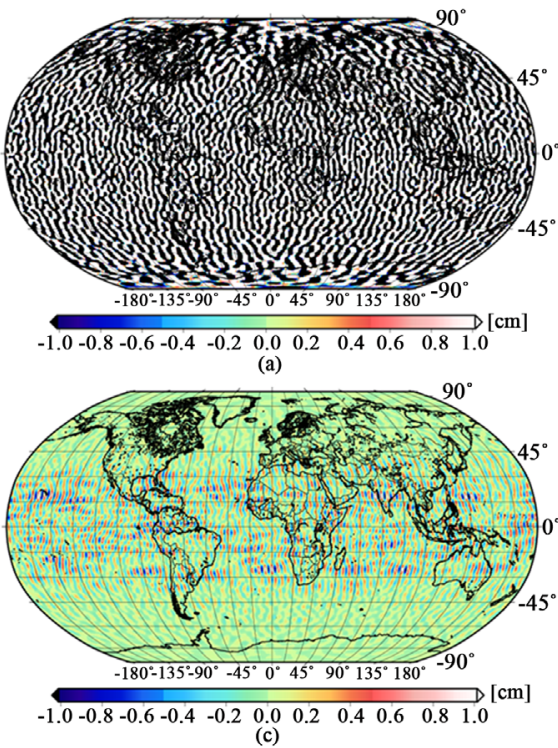

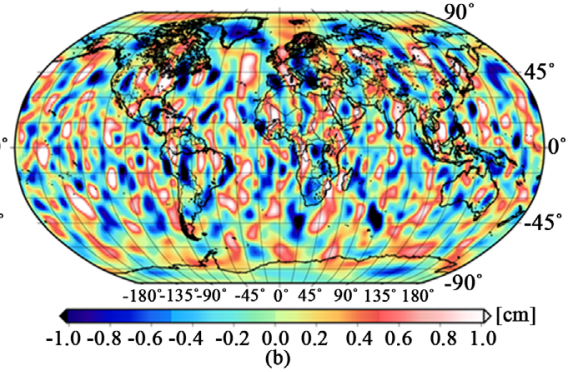

(b)

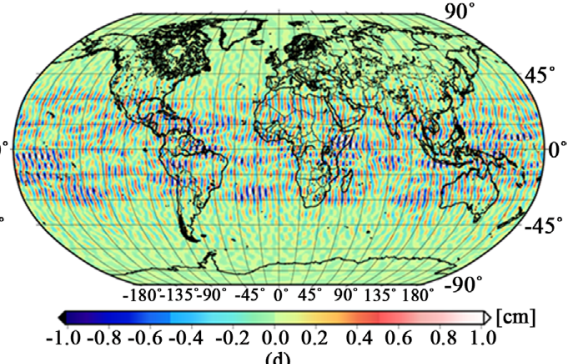

Figure 5. Gravity field solutions in terms of geoid heights $[\mathrm{cm}]$ as determined from the (a) GRACE-like formation; (b) Gaussian filtered (400 km radius) of GRACE-like solution; (c) ST-FGM and (d) PI-FGM.

Table 3. Statistics of the geoid heights $[\mathrm{cm}]$ in terms of standard deviations (SD), mean, minimum (min.) and maximum (max.) values.

\begin{tabular}{ccccc}
\hline \multirow{2}{*}{$\begin{array}{c}\text { Satellite } \\
\text { Formation }\end{array}$} & SD & mean & min. & max. \\
\cline { 2 - 5 } & 13.748 & -0.007 & -76.793 & 74.686 \\
GRACE-like & & & -1.617 & 1.608 \\
GRACE-like & 0.407 & 0.0011 & -1.162 & 1.271 \\
(filtered @ 400 km) & 0.170 & $2.49 \mathrm{e}-05$ & -1.458 & 1.387 \\
ST-FGM & 0.216 & $-7.11 \mathrm{e}-05$ & - & \\
PI-FGM & & & &
\end{tabular}


(Figure 5(d)), an overall SD of about $0.216 \mathrm{~cm}$ (Table 3 ) in terms of retrieved geoid heights provides improvements by a factor of about 63 w.r.t. the GRACE-like formation (Figure 5(a)). In addition, the ST-FGM mission architecture apparently provides the overall least formal errors of the estimated gravity coefficients as shown in Figure 4 (dashed curves) w.r.t. the GRACE-like and PI-FGM mission configurations.

To sum up, the new design of the ST-FGM architecture represented in Figure 1(b) has proved, besides its stable mission architecture in space, that it provides refined retrieval of the global gravity field of the Earth, which was the scope of this paper.

\section{Conclusions}

In this paper, a new design of future space mission architecture, namely ST-FGM, is presented. The orbital parameters of the classical pre-defined two flying satellite-pairs (polar and inclined) (i.e. PI-FGM) have been spatially (using satellite's RAAN angle) and temporally (using satellite's mean anomaly) modified (i.e. ST-FGM). The classical PI-FGM has showed a possible collision risk between its polar and inclined satellite-pairs, whereas, the ST-FGM avoids within its architecture any possible collision risk. For all investigated mission architectures, repeat orbits were fairly selected as identical as possible, which were set to be 11 days in this study. Noisy measurements were used to produce the gravity field parameters in terms of spherical harmonics up to degree/order (d/o) 100/100.

Flying an inclined-pair through the ST-FGM and PI-FGM mission architecture has showed an improvement of about two full orders of magnitude in the spectral domain w.r.t. a single satellite-pair represented by the GRACE-like formation. Moreover, the ST-FGM provided an overall improvement by a factor of about 80 w.r.t. the GRACE-like formation and a factor of about $\approx 2.5$ w.r.t. the smoothed gravity solution using the Gaussian filter at radius $400 \mathrm{~km}$. Additionally, the ST-FGM mission architecture has provided overall least estimated formal errors of w.r.t. the other investigated mission configurations; the "reference" GRACE-like and the classical PI-FGM. Therefore, one worthy recommends here the shifted spatio-temporal polar-inclined (ST-FGM) to be considered as one of stable future architectures of satellite gravity missions.

\section{Acknowledgements}

The author of this paper would like to thank the editor and the anonymous reviewers who improved the quality of this paper. The spatial representations of the results have been plotted using the GMT5 (Generic Mapping Tools) software [26].

\section{Conflicts of Interest}

The author declares no conflicts of interest regarding the publication of this paper. 


\section{References}

[1] Reigber, C., Schwintzer, P. and Lühr, H. (1999) The CHAMP Geopotential Mission. Bolletino di Geofisica Teoricaed Applicata, 40, 285-289.

[2] Tapley, B.D., Bettadpur, S., Watkins, M. and Reigber, C. (2004) The Gravity Recovery and Climate Experiment: Mission Overview and Early Results. Geophysical Research Letters, 31, 1-4. https://doi.org/10.1029/2004GL019920

[3] Kornfeld, R., Arnold, B., Gross, M., Dahya, N. and Klipstein, W. (2019): GRACE-FO: The Gravity Recovery and Climate Experiment Follow-On Mission, Journal of Spacecraft and Rockets, 56. https://doi.org/10.2514/1.A34326

[4] ESA (1999) Gravity Field and Steady-State Ocean Circulation Explorer Mission. Report of European Space Agency for Mission Selection, the Fourth Canditate Earth Explorer Core Missions, SP-1233, Nordwijk, Netherlands.

[5] Sneeuw, N. and Schaub, H. (2005) Satellite Clusters for Future Gravity Field Missions. In: Jekeli, C., Bastos, L. and Fernandes, J., Eds., Gravity, Geoid and Space Missions, Volume 129, Springer, Berlin, Heidelberg, 12-17. https://doi.org/10.1007/3-540-26932-0 3

[6] Bender, P., Wiese, D. and Nerem, R. (2008) A Possible Dual-GRACE Mission with 90 Degree and 63 Degree Inclination Orbits. Third International Symposium on Formation Flying, Europe Space Agency, Noordwijk, Netherlands, 23-25 April 2008.

[7] Sneeuw, N., Sharifi, M. and Keller, W. (2008) Gravity recovery from formation flight missions. In: Xu, P., Liu, J. and Athanasios, D., Eds., VI Hotine-Marussi Symposium on Theoretical and Computational Geodesy, Volume 132, Springer, Berlin, Heidelberg, 29-34. https://doi.org/10.1007/978-3-540-74584-6 5

[8] Wiese, D., Folkner, W. and Nerem, R. (2009) Alternative Mission Architectures for a Gravity Recovery Satellite Mission. Journal of Geodesy, 83, 569-581.

https://doi.org/10.1007/s00190-008-0274-1

[9] Elsaka, B. (2010) Simulated Satellite Formation Flights for Detecting the Temporal Variations of the Earth's Gravity Field. Ph.D. Thesis. University of Bonn, Bonn, Germany.

[10] NG2 Team (2011) Assessment of a Next Generation Gravity Mission to Monitor the Variations of Earth's Gravity Field. Final Report.

[11] NGGM Team (2011) Assessment of a Next Generation Gravity Mission to Monitor the Variations of Earth's Gravity Field. ESA Contract No. 22643/09/NL/AF, Executive Summary. Thales Alenia Space Report SD-RP-AI-0721, March 2011.

[12] Wiese, D., Nerem, R. and Han, S.-C. (2011) Expected Improvements in Determining Continental Hydrology, Icemass Variations, Ocean Bottom Pressure Signals, and Earthquakes Using Two Pairs of Dedicated Satellites for Temporal Gravity Recovery. Journal of Geophysical Research: Solid Earth, 116, 405. https://doi.org/10.1029/2011JB008375

[13] Wiese, D., Nerem, R. and Lemoine, F. (2012) Design Considerations for a Dedicated Gravity Recovery Satellite Mission Consisting of Two Pairs of Satellites. Journal of Geodesy, 86, 81-98. https://doi.org/10.1007/s00190-011-0493-8

[14] Elsaka, B., Kusche, J. and Ilk, K.-H. (2012) Recovery of the Earth's Gravity Field from Formation-Flying Satellites: Temporal Aliasing Issues. Advances in Space Research, 50, 1534-1552. https://doi.org/10.1016/j.asr.2012.07.016

[15] Elsaka, B., Raimondo, J.-C., Brieden, P., Reubelt, T., Kusche, J., Flechtner, F., Iran Pour, S., Sneeuw, N. and Müller, J. (2014) Comparing Seven Candidate Mission 
Configurations for Temporal Gravity Retrieval through Full-Scale Numerical Simulation. Journal of Geodesy, 88, 31-43. https://doi.org/10.1007/s00190-013-0665-9

[16] Daras, I., Pail, R., Murböck, M. and Yi, W. (2014) Gravity Field Processing with Enhanced Numerical Precision for LL-SST Missions. Journal of Geodesy, 89, 99-110. https://doi.org/10.1007/s00190-014-0764-2

[17] Iran-Pour, S., Sneeuw, N., Daras, I., Pail, R., Murböck, M., Gruber, T., Tonetti, S., Cornara, S., Weigelt, M., Van Dam, T., Visser, P. and da Encarnação, T. (2015) Assessment of Satellite Constellations for Monitoring the Variations in Earth Gravity Field-SC4MGV. ESA-ESTEC Contract No. AO/1-7317/12/NL/AF, Final Report.

[18] Flechtner, F., Neumayer, K.H., Dahle, C., Dobslaw, H., Fagiolini, E., Raimondo, J.C. and Guntner, A. (2016) What Can be Expected from the GRACE-FO Laser Ranging Interferometer for Earth Science Applications? Surveys in Geophysics, 37, 453-470. https://doi.org/10.1007/s10712-015-9338-y

[19] Pail, R., Gruber, T., Abrykosov, P., Hauk, M. and Purkhauser, A. (2018) Assessment of NGGM Concepts for Sustained Observation of Mass Transport in the Earth System. GRACE/GRACE-FO Science Team Meeting, Potsdam, Germany, 10 October 2018. https://meetingorganizer.copernicus.org/GSTM-2018/GSTM-2018-10.pdf

[20] Nie, Y., Shen, Y. and Chen, Q. (2019) Combination Analysis of Future Polar-Type Gravity Mission and GRACE Follow-On. Remote Sensing, 11, 200. https://doi.org/10.3390/rs11020200

[21] Rees, W.G. (2001) Physical Principles of Remote Sensing. 2nd Edition, Cambridge University Press, Cambridge, United Kingdom.

[22] Kaula, W.M. (1966) Theory of Satellite Geodesy. Blaisdell Publishing Company, Waltham, MA.

[23] Mayer-Gürr, T. (2006) Gravitationsfeldbestimmung aus der Analyse kurzer Bahnbögen am Beispiel der Satellitenmissionen CHAMP und GRACE. Ph.D. Thesis, University of Bonn, Bonn.

[24] Mayer-Gürr, T., Eicker, A., Kurtenbach, E. and Ilk, K.H. (2010) ITG-GRACE: Global Static and Temporal Gravity Field Models from GRACE Data. In: Flechtner, F., Gruber, T., Gntner, A., Mandea, M., Rothacher, M., Schne, T. and Wickert, J., Eds., System Earth via Geodetic-Geophysical Space Techniques, Springer, Berlin, 159-168. https://doi.org/10.1007/978-3-642-10228-8 13

[25] Jekeli, C. (1981) Alternative Methods to Smooth the Earth's Gravity Field. The Ohio State University, Columbus, $\mathrm{OH}$.

[26] Wessel, P., Smith, W.H.F., Scharroo, R., Luis, J.F. and Wobbe, F. (2013) Generic Mapping Tools: Improved Version Released. Eos, Transactions, American Geophysical Union, 94, 409-410. https://doi.org/10.1002/2013EO450001 